\title{
Design and Evaluation of Bamboo-Based Elastic Cushion by Human Pressure Distribution
}

\author{
Zheng Jipenga ${ }^{\mathrm{a}}$, Zhi Zhenhao ${ }^{\mathrm{a}}$, Zhang Enchang, ${ }^{\mathrm{a}, \mathrm{b}}$, Yu Yuxiang ${ }^{\mathrm{a}, \mathrm{b}, 1}$ and Wu Qun \\ ${ }^{a}$ School of Art and Design, Zhejiang Sci-Tech University \\ ${ }^{\mathrm{b}}$ Lab of Material Innovation and Intelligent Interaction, Zhejiang Sci-Tech University
}

\begin{abstract}
Bamboo waste, mostly from the processing of bamboo products, can be used as raw materials to prepare the elastic cushion. The bamboo-based elastic cushion (BEC) units were firstly designed through mixing low melting-temperature fibers based on human pressure distribution. And the influence of heating temperature, heating time and bamboo proportion on the elastic modulus of BEC units was investigated. Then, the BEC was prepared by the permutation and combination of BEC units with different elastic modulus and its comfort level was ranked by subjective evaluation. Results showed that heating temperature, heat time and bamboo proportion all had clear influence of elastic modulus of BEC units, and its order of influence intensity was bamboo proportion, heating temperature and heating time. Subjective evaluation indicated that the modular design method based on human pressure distribution could effectively improve the comfort of BEC. These results indicated that the BEC prepared with bamboo waste fibers and ES fibers by human pressure distribution had good mechanical properties and comfort.
\end{abstract}

Keywords. Bamboo waste, Elastic cushion, Human pressure distribution, Design and evaluation

\section{Introduction}

Bamboo can be used as an alternative material to wood because of its fast growth rate and short growth cycle [1]. However, the current utilization rate of bamboo is only $40 \%$, and the remaining bamboo waste is usually handled as fuel of low value [2]. Therefore, it is necessary to find a way to improve the efficient recycling of bamboo waste. With the development of science and technology, bamboo waste has been gradually applied to prepare bamboo-based artificial board, such as bamboo plywood, bamboo particle board, and bamboo fiberboard. In addition, some researchers used bamboo waste to prepare bamboo-based elastic materials, and found that had good air permeability and resilience, strong strength in longitudinal and transverse [3]. This indicates that bamboo waste has excellent prospects for applying in the fried of elastic fillers in cushions.

With the improvement of living standards, the requirements for cushions became greener and more renewable, thus increasing the research on applying natural material to

\footnotetext{
${ }^{1}$ Corresponding Author, Yuxiang Yu, Lab of Material Innovation Design and Intelligent Interaction, Zhejiang Sci-Tech University, 928 Seconded Avenue, Xiasha High Education Zone, Hangzhou 310018, China, yuyuxiang0612@zstu.edu.cn.
} 
produce elastic cushions, such as, loofah, monofilament, etc. [4]. Comfort, one of the most important evaluation indicators of elastic cushions, was influenced by the shape, material and craftmanship. Besides, Cushion comfort is also related on the distribution of human pressure [5-7]. The human hip is the part having the largest contact area with the cushion, so the force on the hip is the most direct factor affecting the comfort lever [8]. Allocating the hardness of different areas of the cushion according to the human pressure distribution, can optimize the force on the hip so as to improve the comfort level [9-10]. To evaluate the comfort level of elastic cushions, subjective evaluation is a commonly used way. Cheng et al. used this method to evaluate the comfort of airplane seats. Xu et al. analyzed the effect of sponge density on cushion comfort by the method of subjective evaluation, found the optimal foam density for the cushion [11-14].

In order to efficiently use bamboo waste and improve comfort level of cushions, the design method of the elastic cushion of bamboo waste (BEC) was proposed based on the human pressure distribution. The influence of heating temperature, heating time and mixing ratio of bamboo waste and low melting-temperature fibers $(\mathrm{B} / \mathrm{BEC}$ ratio) on the elastic modulus of BEC units was investigated, and the comfort level of BEC was ranked by subjective evaluation. The results provide a scientific and theoretical basis for the development and design of bamboo-based products, and provide a reference basis for the development and design of other natural fiber material cushions.

\section{Method}

\subsection{Pressure distribution}

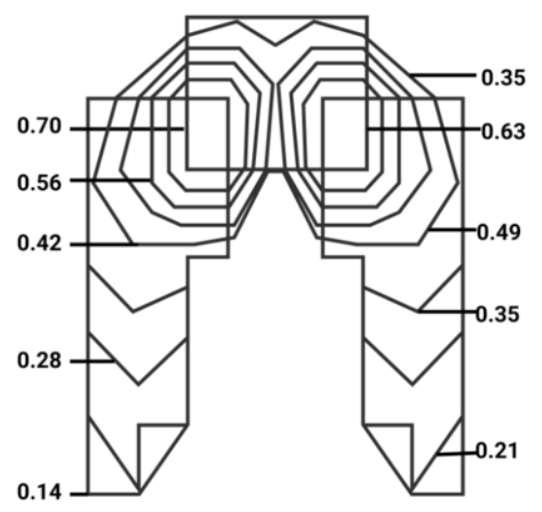

Figure 1. Ideal pressure distribution of human body [16]

Based on human physiology and anatomy, the ideal body pressure distribution is shown in Fig 1. The pressure value of sciatic node is the biggest, and the minimum pressure value appears at the contact part between thigh and front end of seat. In the posterior femoral region, the pressure value tends to decrease gradually. The overall pressure distribution should be symmetrical from left to right, and there should not have a peak pressure value that makes the person feel foreign [15]. Therefore, to reach the ideal body pressure distribution, the location of sciatic node needs to be set with an elastic material with high hardness to give sufficient support. The hardness is mainly related to the elastic 
modulus and thickness of elastic material in the cushion. Because the thickness is difficult to change due to the predefined size and structure of cushion, controlling the elastic modulus of BEC is a more effective method to realize the goal of ideal body pressure distribution.

\subsection{Modular partition}

The existing cushions are poorly comfortable due to the whole filling. These cushions are easy to cause deformation and collapse of contact pressure area after using for a long time, thus enhancing the probability of disease because of the obstruction of blood flow. By modular design method, the cushion was divided into several tightly combined elastic units, and then the elastic modulus of the units could be arranged according to different pressure, so that the cushion could fit the hip and made the user get a comfortable experience.

\subsection{Subjective evaluation}

Subjective evaluation can directly and clearly reflect the comfort of cushion. Users or subjects, as non-professional designers, could not give an accurate comfort evaluation. But they can give clear feedback on the comfort from a critical part of body. Therefore, the key point to evaluate the comfort of cushion is the comfort from the critical parts of human body [17]. In order to verify whether the arrangement of BEC units that fit the human pressure distribution is effective, a control group should be set up for subjective evaluation test. The size, dimensions, and unit number of control group cushion should be consistent with those of the experimental group.

\section{Design Practice}

\subsection{Preparation and characterization of BEC units}

The bamboo waste with 18 24\% moisture content was provided by Tonglu Yunfeng Bamboo Products Factory, Zhuji City, Zhejiang Province, China. The ethylenepropylene side by side (ES fiber) was bought from Jiangsu Yufun New Material Technology Co., Ltd. The yellow bamboo fiber (width $3 \sim 5 \mathrm{~mm}$ and unfolded length $180 \sim 200 \mathrm{~mm}$ ) was selected from bamboo waste and dried at $108 \pm 2{ }^{\circ} \mathrm{C}$ for $24 \mathrm{~h}$. Then the dried yellow bamboo fiber was mixed with the ES fiber at different mixing ratio. 20 g mixture was added into a square metal mold with a side length of $60 \mathrm{~mm}$, then put into the drying oven at different temperature and time to prepare the BEC units. The pressure distribution on the human hip needs to be changed by adjusting the material elastic modulus of bamboo waste when designing BEC. When bamboo fibers were bonded with low melting point fibers by heating, the performance was mainly related to the content of bamboo waste [3], so the $\mathrm{B} / \mathrm{BEC}$ ratio needs to be changed to prepare the BEC units with different elastic modulus. The elastic modulus of BEC units were tested by EN 15534-1-2014. 
Table 1. Factors level of BEC units

\begin{tabular}{cccc}
\hline \multirow{2}{*}{ Level } & $\begin{array}{c}\text { Factor 1 } \\
\text { Heating temperature }\left({ }^{\circ} \mathrm{C}\right)\end{array}$ & $\begin{array}{c}\text { Factor 2 } \\
\text { Heating time (min) }\end{array}$ & $\begin{array}{c}\text { Factor 3 } \\
\text { Bamboo proportion (\%) }\end{array}$ \\
\hline 1 & 130 & 30 & 40 \\
2 & 140 & 40 & 45 \\
3 & 150 & 50 & 50 \\
4 & 160 & 60 & 55 \\
5 & & & 60 \\
6 & & & 65 \\
7 & & & 70 \\
8 & & & 75 \\
9 & & & 80 \\
\hline
\end{tabular}

\subsection{Preparation and evaluation of $B E C$}

The BEC consisted of $64 \mathrm{BEC}$ units with an $8 \mathrm{X} 8$ arrangement. The BEC units were divided into five grades according to the elastic modulus values, and the ideal human pressure distribution was also divided into five grades according to the pressure value. As a reference, the pressure of sciatic nodes corresponded to the high-grade elastic modulus units. Then BEC units were arranged based on the elastic modulus grade in accordance with the pressure distribution diagram of human sitting position. The arranged BEC units was covered both side with a layer of $1.5 \mathrm{~mm}$ low-melting fiber cloth, and put in a $60 \mathrm{~mm}$ high metal mold with adjustable length and width, and heated at $150{ }^{\circ} \mathrm{C}$ under normal pressure for $10 \mathrm{~min}$ to obtain the $\mathrm{BEC}$, named $\mathrm{BEC} 1$. To verify the comfort of the cushion, a cushion consisting of 64 uniformly BEC units with $65 \%$ bamboo proportion was prepared as a comparison, named BEC2.

To verify the comfort of $\mathrm{BEC}$ according to the human pressure distribution, 10 subjects were invited to conduct a subjective evaluation experiment for comfort. All subjects were adults whose height and weight were between 5th and 95th percentile of the standard Chinese adult body size. The basic information of subjects is shown in Tab.2.

Table 2. Basic information of subjects

\begin{tabular}{ccccc}
\hline No. & Gender & Height $\mathbf{( c m})$ & Weight $\mathbf{( k g )}$ & Age \\
\hline 1 & Female & 163 & 57 & 23 \\
2 & Male & 176 & 67 & 24 \\
3 & Male & 176 & 72 & 24 \\
4 & Female & 158 & 47 & 24 \\
5 & Male & 179 & 75 & 23 \\
6 & Female & 160 & 46 & 25 \\
7 & Female & 161 & 53 & 22 \\
8 & Male & 170 & 65 & 23 \\
9 & Female & 155 & 45 & 24 \\
10 & Male & 177 & 75 & 26 \\
\hline
\end{tabular}

In order to assess more accurately on the comfort level of different parts of body while sitting on the cushion, the key body parts were selected as follows: hips, sciatic nodes, thighs, and thigh roots. The evaluation scale was divided into 7 levels according to the comfort level, with 1 is very uncomfortable, 2 is uncomfortable, 3 is somewhat uncomfortable, 4 is average, 5 is okay, 6 is comfortable, and 7 is very comfortable. The 
testing steps were as follows: the subjects sat on the BEC in an upright position for 5 mins, then rated the comfort level of the corresponding body parts.

\section{Results and discussion}

\subsection{Elastic modulus}

The influence of heating temperature and heating time on elastic modulus of BEC units is displayed in Fig.2. As shown in Fig.2a, with the increase of heating temperature, the elastic modulus of BEC units gradually increased. The melting point of ES fibers is $130{ }^{\circ} \mathrm{C}$. When the heating temperature rase above $130{ }^{\circ} \mathrm{C}$, more ES fibers were in a molten state to bonding bamboo waste fibers as an adhesive. High heating temperature could improve the bonding strength between bamboo waste fibers and ES fibers, thus increasing the elastic modulus of BEC units.
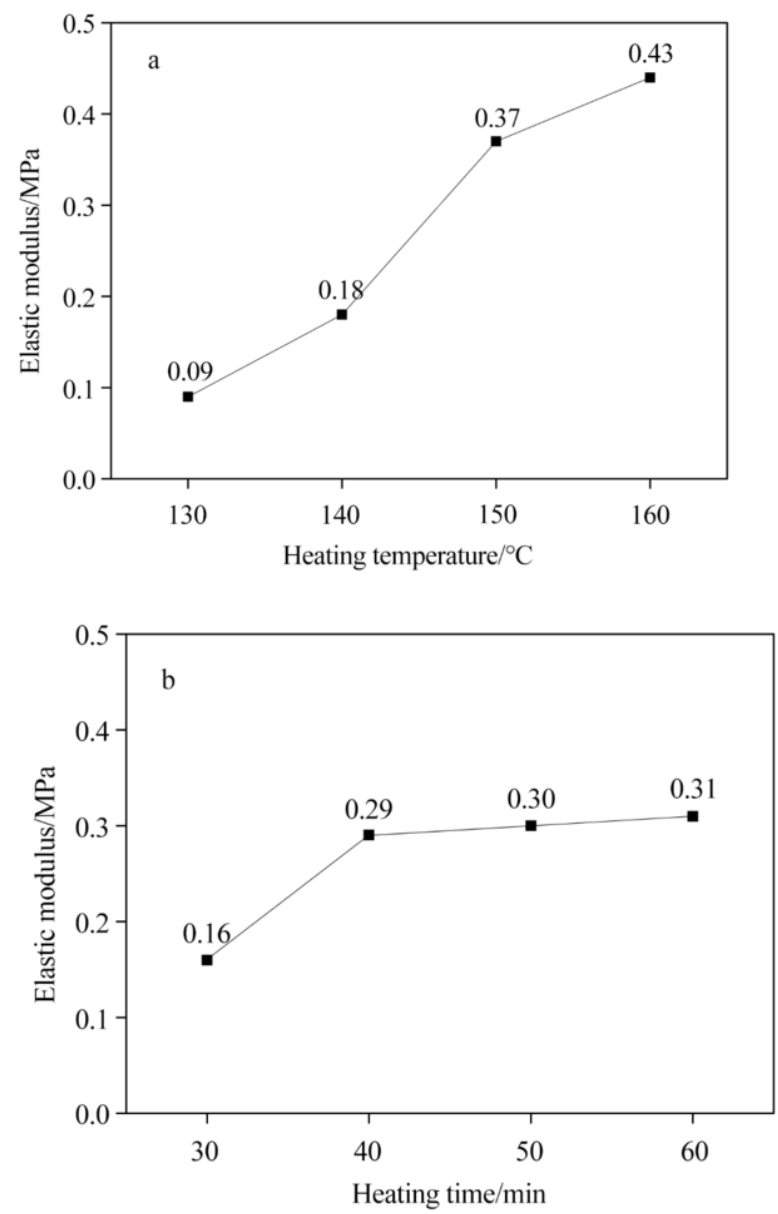

Figure 2. Influence of heating temperature, and heating time on elastic modulus of BEC units 
As seen in Fig.2b, with the increasing heating time, the elastic modulus of BEC units increased first and then tended to be stable after the heating time reaching $40 \mathrm{~min}$. The size of BEC units was lager, so the heat needed more time to transfer from the surface to inner core. Fig. 3 shows the combination of bamboo waste and ES fiber inside the BEC units. As shown in Fig 3, the BEC units were cut from the middle part, and with the increase of heating time, the bamboo waste fibers inside the unit gradually bonded with the ES fibers, and bonded completely at $50 \mathrm{~min}$. These indicated that there was no direct relation between the change of elastic modulus and bonding situation of inner core of BEC units. But, considering that the incomplete bonding of inner core might lead to the collapse of BEC units, the heating time should be longer than 50min. Compare with the influence of heating temperature and heating time, the effect of heating temperature on the elastic modulus of BEC units was more significant than heating time.

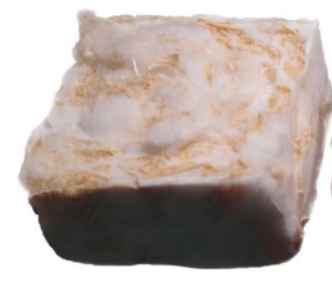

30

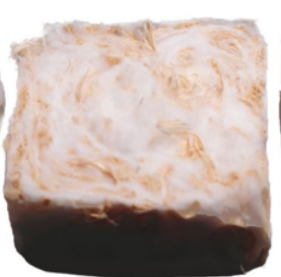

40

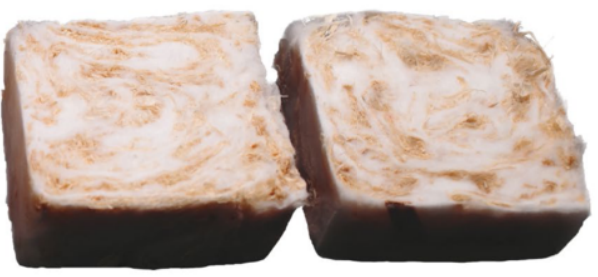

50

60

Heating time/min

Figure 3. Combination of bamboo waste fibers and ES fibers inside the BEC units.

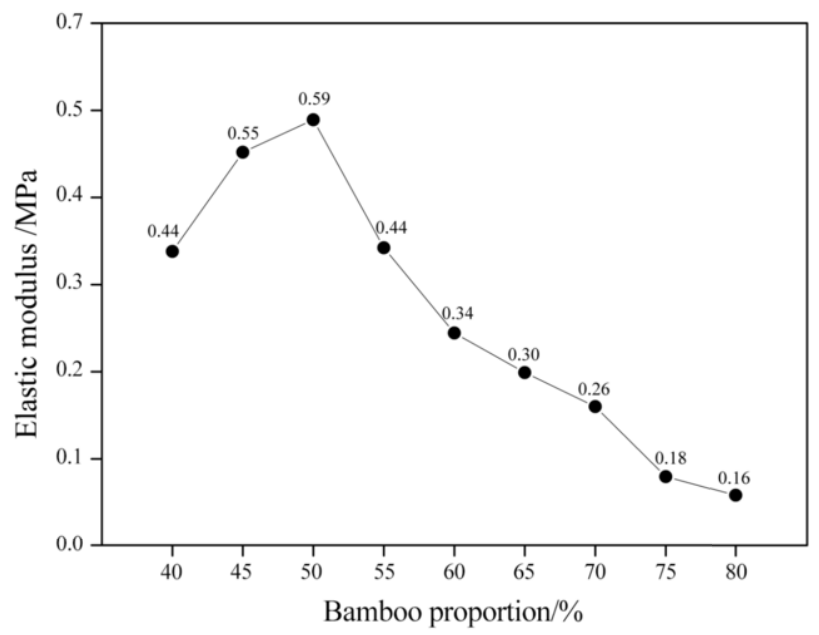

Figure 4. Influence of bamboo proportion on elastic modulus of BEC units

The influence of bamboo proportion on elastic modulus of BEC units is displayed in Fig. 5. As seen in Fig.5, with the increasing bamboo proportion, the elastic modulus of BEC units increased first, and then decreased, reaching the maximum at $50 \%$ bamboo proportion. The bamboo waste fibers provided the elastic for BEC units, and the ES 
fibers provided the bonding strength. When the bamboo proportion was below $50 \%$, there were no enough bamboo waste fibers to support the BEC units even with the excellent bonging inside. Oppositely, when the bamboo proportion was over $50 \%$, the bonding strength between bamboo waste fibers and ES fibers began decreasing, which would also decrease the elastic modulus of BEC units.

The higher bamboo proportion meant the better sustainability of BEC units. Thus, more BEC units with high bamboo proportion were prepared. The elastic modulus of BEC units obtained from different bamboo proportion could be divided into five classes and matched with the human sitting pressure: $55 \%$ for $0.40 \sim 0.45 \mathrm{MPa}, 60 \%$ for $0.35 \sim 0.40 \mathrm{MPa}, 65 \%$ for $0.30 \sim 0.35 \mathrm{MPa}, 70 \%$ for $0.25 \sim 0.30 \mathrm{MPa}, 75 \%$ for $0.20 \sim 0.25$ $\mathrm{MPa}$. Based on the design method of BEC based on human pressure distribution, the bamboo waste elastic unit was arranged according to the above-mentioned matching method, and the arrangement results were shown in Fig 5.
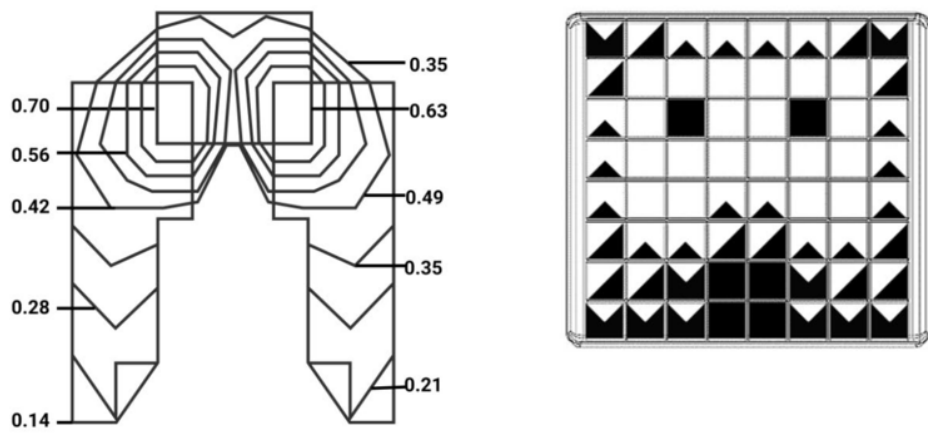

Elastic modulus/ MPa

$0.18 \sim 0.20$

$0.20 \sim 0.22$

$0.22 \sim 0.24$

$0.24 \sim 0.26$

$0.26 \sim 0.28$

Figure 5. Schematic diagram of cushion modular distribution and elastic modulus grades

\subsection{Subjective evaluation}

Table 3. Average comfort score of key parts (Standard deviation in parentheses)

\begin{tabular}{cccccc}
\hline Cushion & Hips & Sciatic nodes & Thighs & Thigh roots & Total comfort \\
\hline \multirow{2}{*}{ BEC1 } & 4.9 & 4.8 & 4.5 & 4.4 & 4.7 \\
& $(0.94)$ & $(0.75)$ & $(0.81)$ & $(0.66)$ & $(0.64)$ \\
BEC2 & 2.5 & 2.9 & 2.7 & 2.9 & 2.8 \\
& $(0.81)$ & $(0.70)$ & $(1.00)$ & $(1.04)$ & $(0.60)$ \\
\hline
\end{tabular}

The average comfort score of key parts on human body is shown in Tab 3. The BEC1 had a higher comfort value than that of BEC2, which indicated that the modular design method based on human pressure distribution could effectively improve the comfort of BEC. Besides, the comfort values on the hip and sciatic node of BEC1 were higher than those for the leg, showing that the unit arrangement in BEC1 provided sufficient support to the sciatic node and reduced the pressure on the hip. The comfort value on the hip of BEC2 was lower than those for the sciatic node and leg area because the unit of BEC2 was stiffer than that of BEC1. The stiff units could provide support while simultaneously compress the hip and thigh muscles, resulting in poor comfort. The results showed that the BEC2 with the uniform arrangement of units could not effectively disperse the pressure on each part of the body, but the $\mathrm{BEC} 1$ arranged according to the body pressure could effectively disperse the pressure while supporting the body, improving the use comfort. 


\section{Conclusion}

(1) Heating temperature, heat time and bamboo proportion all had clear influence of elastic modulus of BEC units. The order of influence intensity was bamboo proportion, heating temperature and heating time.

(2) The subjective evaluation indicated that the modular design method based on human pressure distribution could effectively improve the comfort of BEC. The pressure of other key parts of the body could be dispersed while the sciatic nodes got enough support.

(3) The BEC units prepared with bamboo waste fibers and ES fibers had good mechanical properties and comfort by human pressure distribution.

\section{Acknowledgments}

The financial support for this work was from the Philosophy and Social Science Planning Project of Zhejiang Province (No. 21NDQN234YB), Humanities and Social Sciences Youth Fund of Ministry of Education

(Research on Chinese Traditional Fiber Product Design Strategy Based on DFS Theory, No. 20YJC760037), and Talent Introduction Program of Zhejiang Sci-Tech University (No. 19082425-Y and 19082153-Y).

\section{References}

[1] Shi, J.Y., et al, The History and Current Situation of Resources and Development Trend of the Cultivated Bamboos in China, Acta Horticulturae, no. 1035, (2014): 71-78.

[2] Zheng Long, Wu Yiqiang, and Zuo Yingfeng, Research Status and Prospects of Residual Use of Bamboo Residues, World Forestry Research 34.03(2021):82-88.

[3] Liang Jiaqi, et al, Research on Properties of Bamboo Fiber/Low-melting-point Polyester Composite Felt, Advanced Textile Technology 28.02(2020):14-19.

[4] Chen Biqi, and Guan Hui yuan, Research and Application of Loofah Cushion Filling Material Based on Resource Utilization of Plant Fiber, Furniture 42.04(2021):16-20+35.

[5] Li Juan, et al, Seat Comfort Characterization by Body-seat Interface Pressure Distribution, Mechanical Science and Technology for Aerospace Engineering 33.09(2014):1298-1303.

[6] Liu Tao, Research on Methodology of Seat Comfort Design Based on Body Pressure Distribution, Jilin University, MA thesis, 2015.

[7] Jin Xiaoping, et al, An Objective Evaluation Method for the Comfort of Foam Cushion in Vehicle Seat, Automotive Engineering 34.06(2012):551-555+565.

[8] Zacharkow, Dennis, Posture: Sitting, Standing, Chair Design, and Exercise, Charles C. Thomas, 1988.

[9] Dong Ming, and Dai Shanqi, Comfort Analysis of Automobile Seats Based on Foaming Hardness Distribution, Auto Engineer, 07(2019):30-33.

[10] Zhang Zhifei, et al, Custom-contoured Cushion Interface Design Method Based on Interface Pressure Mapping, Journal of mechanical engineering 55.17(2019):162-171.

[11] Annett, John, Subjective Rating Scales: Science or Art? Ergonomics, vol.45, no.14,(2002): 966-987.

[12] Cheng Peng, Analysis and Optimization of Aircraft Seat Comfort Based on Static Body Pressure Distribution, Hunan University, MA thesis, 2020.

[13] Xu Wei, et al, The Influence of Sponge Density on the Comfort of Sofa Cushions, Furniture, 39.04(2018): 17-21+46.

[14] Liang Ling, Research on the Comfort of Foam Cushion for Vehicle Seat, Chongqing University, MA thesis, 2018.

[15] Wang Lina, Research on the Comfort of High-Speed Rall Chair Surface Based on Spd, Donghua University, MA thesis, 2017.

[16] Bai Shiqi, The Study of Cushion Pressure Distribution Based on Ergonomics, Zhejiang Sci-Tech University, MA thesis, 2014.

[17] Hou Jianjian, Zhang Xuerong, and Ren Lihui, Simulation Analysis of Vehicle Seat Seating Reference Point and Pressure Distribution, Modern Transportation Technology, 8.04(2011):65-67. 